\title{
Pollen morphology and its taxonomic significance in the genus Bomarea Mirb. (Alstroemeriaceae) - II. Subgenus Bomarea
}

Abul Khayer Mohammad Golam Sarwar ${ }^{1,2 \star}$, Yoichiro Hoshino ${ }^{1}$ and Hajime Araki $^{1}$

Received: July 15, 2015 Accepted: September 24, 2015

\begin{abstract}
Pollen morphology of 52 species (out of $c$. 79) of the Bomarea subgenus Bomarea was examined using light microscopy and scanning electron microscopy (SEM), or using SEM alone. The studied species of Bomarea were stenopalynous, characterized by large, oblate, monosulcate monads with reticulate exine sculpture in most species. Wide variation was observed in quantitative palynological features. The studied taxa were divided into four major groups based on exine ornamentation observed under SEM: microreticulate, reticulate, coarsely rugulate, and psilate-perforate. The reticulate exine sculpture may be a plesiomorphic character state for the genus Bomarea, and the coarsely rugulate and finely rugulate-perforate or psilate-perforate exine sculptures may have evolved independently more than once. In agglomerative hierarchical clustering (AHC) analyses of the genus Bomarea using quantitative pollen data, the studied species were distributed in either two (similarity-based) or four (dissimilarity-based) major clusters. Neither the recent molecular phylogenetic analyses nor the AHC analyses of Bomarea have recovered clades/clusters that represent traditionally recognized subgeneric taxa for the genus. Therefore, the most reliable infrageneric classification of Bomarea can be achieved by combining morphological, palynological, and molecular data from more extentive sampling of all the species.
\end{abstract}

Keywords: agglomerative hierarchical clustering, Bomarea, exine sculpture, infrageneric classification, scanning electron microscopy

\section{Introduction}

Bomarea is the most diverse genus of Alstroemeriaceae, and it includes 100-120 species with a Neotropical distribution from Mexico in the north to Argentina/Chile in the south (Neuendorf 1977; Alzate 2005). The genus is largely restricted to the Andean range and its continuation in Central America (Hofreiter \& Tillich 2002). Based on morphological features, the genus Bomarea is divided into four subgenera: Baccata (five spp.), Bomarea s. str. (c. 79 spp.), Sphaerine (12 spp.), and Wichuraea (18 spp.; Hofreiter \& Tillich 2002). The subgenus Bomarea is characterized by a generally twining habit, with flowers pendulous and actinomorphic or horizontally oriented and zygomorphic, tepals deciduous, ovary inferior and dehiscent, and fruit leathery. Baker (1888) divided this subgenus into four main groups according to the structure of the inflorescence and the relation of the inner and outer tepals, and Killip (1935) later named these four groups Multiflorae, Caldasianae, Edules, and Vitellinae. Presently, four sections are recognized within the subgenus Bomarea: Multiflorae, Edules, Goniocaulon, and Pardinae (Harling \& Neuendorf 2003; for details see Hofreiter 2008).
As part of a comprehensive survey of pollen morphology in the genus Bomarea, pollen studies of three subgenera, viz. Baccata, Sphaerine, and Wichuraea, have already been published (Sarwar et al. 2015 and references therein). Light microscopy (LM) was mainly employed in previous palynological studies of this genus, and scanning electron microscopy (SEM) was employed in a few cases (for details see Sarwar et al. 2015). We describe here, using both LM and SEM, the pollen morphological features of the subgenus Bomarea, and evaluate their usefulness for the infrageneric classification of this genus.

\section{Materials and Methods}

Pollen morphology of 52 species (out of c. 79) of the Bomarea subgenus Bomarea and of one species each of the subgenera Baccata and Wichuraea was examined by light microscopy (LM) and scanning electron microscopy (SEM), or by SEM alone (Tab. 1). Polliniferous materials used in this investigation were taken from dried specimens from the herbaria K, MO, MOL, NY, and USM. Herbarium abbreviations follow the Index Herbariorum (Thiers 2007).

\footnotetext{
${ }^{1}$ Field Science Center for Northern Biosphere, Hokkaido University, Sapporo, Japan.

${ }^{2}$ Present address: Department of Crop Botany, Bangladesh Agricultural University, Mymensingh 2202, Bangladesh.

*Corresponding author: drsarwar@bau.edu.bd
} 
Table 1. List of Bomarea taxa used in this study along with their voucher specimens. ${ }^{*}$ New palynological data.

\begin{tabular}{|c|c|c|}
\hline No. & Taxa & Voucher specimens \\
\hline 1. & B. acutifolia (Link \& Otto) Herb. & Costa Rica: Prov. San Jose-Cartago, Cordillera de Talamanca, Davidse 24682 (MO3135888) \\
\hline 2. & B. amazonica Hofr. \& Rod.* & Peru: Prov. Bagua, La Peca, Humid cloud forest, 12.08.1978. Barbour 2859 (MO2796435) \\
\hline 3. & B. anceps (R. \& P.) Herb.* & Peru: (MOL2940370) \\
\hline 4. & B. andreana Baker* & $\begin{array}{l}\text { Costa Rica: Prov. San Jose, northern Cordillera Talamanca, Cerro da la Muerte, 04.04.1978. Davidson } 7239 \\
\text { (MO2660647) } \\
\text { Panama: Chiriqui, Boquete, } 18.05 .1976 . \text { Croats } 34872 \text { (MO2698093) }\end{array}$ \\
\hline 5. & B. angulata Benth.* & Peru: Lambayeque, Ferrenafe, 11.09.1985. Sagastegui 12759 (MO3328919) \\
\hline 6. & B. aurantiaca Herb.* & $\begin{array}{l}\text { Bolivia: Cochabamba, Chapare, 17.02.1971. Hawkes et al. } 4438 \text { (MO2888570) } \\
\text { Peru: Depto. Cusco, Prov. Paucartambo. 03.15.1991. Cano } 4679 \text { (USM107269) }\end{array}$ \\
\hline 7. & B. boliviensis Baker & $\begin{array}{l}\text { Bolivia: Chuguisaca, Sucre, 2.1.1994. Wood } 7818(\mathrm{~K}) \text {; } \\
\text { La Paz, Murillo, 09.01.1982. Solmon } 6654 \text { (MO3148134) }\end{array}$ \\
\hline 8. & B. bredemeyerana (Schlecht.) Herb. ${ }^{*}$ & Venezuela: Lara, 24.06.1979. Liesner et al. 7935 (MO2994103) \\
\hline 9. & B. campanularia Harl. \& Neuend. & Ecuador: Pichincha, Quito, 08.02.1990. Cerón \& Iguago 8599 (MO04778566) \\
\hline 10. & B. campylophylla Killip* & $\begin{array}{l}\text { Bolivia: La Paz, Franz Tamayo, Senda Apolo-San Jose de Uchupiamonas, 05.12.2002. Miranda et al. } 588 \\
\text { (MO5836245) }\end{array}$ \\
\hline 11. & B. carderi Mast. & Panama: Cocle, 24.11.1983. Churchill 3904 (MO3198551) \\
\hline 12. & B. chiriquina Killip* & Panama: Chiriquí, 20.04.1975. Mori \& Kullunki 5651 (MO3155731) \\
\hline 13. & B. cochabambensis Killip* & Bolivia: Cerveceria Taquina, N-W of Cochabamba, 28.01.1943. Cutler 7678 (NY) \\
\hline 14. & B. cordifolia (R. \& P.) Herbert.* & Peru: Depto. Pasco, Prov. Oxapampa. 9.10.1982. Foster \& Smith 9076 (USM60834) \\
\hline 15. & B. cornigera Herb.* & Peru: Cajamarca, Jaén, 20.02.1985. Stein \& Todzia 2230 (MO3294282) \\
\hline 16. & B. crassifolia Baker & Peru: Cajamarca, Jaén, 10.06.1947. Fosberg 27845 (MO2603296) \\
\hline 17. & B. crocea (R. \& P.) Herb. & Peru: Cusco, Cusco, 23.10.2004. Galiano et al. 6987 (MO04840135) \\
\hline 18. & B. cornuta Herb.* & Peru: Huánuco, Huánuco, 02.03.1985. Stein \& Todzia 2275 (MO3294279) \\
\hline 19. & B. costaricensis Kranz.* & Costa Rica: Cartago, El Guarco, 02.02.1985. Chavarria 663 (MO5850981) \\
\hline 20. & B. densiflora Herb.* & $\begin{array}{l}\text { Peru: Amazonas, Chachapoyas, no day.12.1962. Woytkowski } 7829 \text { (MO1793882) } \\
\text { Junín, 06.11.1962. Vigo } 6229 \text { (MO5384465) }\end{array}$ \\
\hline 21. & B. dolichocarpa Killip ${ }^{*}$ & Peru: Madre de Dias, 25.1.1989. Smith et al. 1579 (K) \\
\hline 22. & B. dispar Herb.* & Peru: Depto. Pasco, Prov. Oxapampa. 30.03.1984. Smith 6582 (USM155639). \\
\hline 23. & B. edulis (Tussac) Herb. & $\begin{array}{l}\text { Japan: Hokkaido, Sapporo, Hokkaido univ., cult. FSC, 15.04.2009. Sarwar \& Hoshino s.n. (SAPS) (LM, SEM) } \\
\text { Mexico: Oaxaca, Ixtlan, 03.07.1988. Luna \& Martin } 0302 \text { (MO4059183) }\end{array}$ \\
\hline 24. & B. endotrachys Kraenzl.* & Peru: Depto. de Pasco, Prov. Oxapampa. 10.12.2002. Vasquez \& Rojas 27762 (USM185591) \\
\hline 25. & B. euryphylla Harl. \& Neuend.* & Ecuador: Napo Quijos, Valle del Rio Cosanga, 19.10.1990. Palacios 6420 (MO04833022) \\
\hline 26. & B. formosissima (R. \& P.) Herb.* & Peru: Cusco, Paucartambo, 30.10.1987. Nunez et al. 8511 (MO3677097) \\
\hline 27. & B. goniocaulon Baker* & Peru: Depto San Martin, 1.9.1965. Hamilton \& Holligan 522 (K) \\
\hline 28. & B. hartwegii Baker* & Peru: Depto. Cajamarca, Prov. San Ignacio. 18.11.1998. Campos et al. 5735 (USM165038) \\
\hline 29. & B. hirsuta (Kunth) Herb. & Costa Rica: San José, Dota, 09.03.1995. Chavarria 756 (MO5850982) \\
\hline 30. & B. hirtella (Kunth) Herb. & Mexico: Chiapas, La Concordia, 15.07.2005. Chagala 68 (MO04854601) \\
\hline 31. & B. lopezii Hofreiter \& E. Rodr.* & Peru: Cajamarca, Contumaza, 20.04.1984. Sagastegui 11402 (MO3226570) \\
\hline 32. & B. lutea Herb.* & Ecuador: Azuay, 10.12.1984. Scolnik 1448 (MO3155643) \\
\hline 33. & B. macusanii Hofreiter \& E. Rodr.* & Peru: Cusco, Paucartambo, 30.10.1987. Nunez et al. 8477 (MO3677094) \\
\hline 34. & B. martiana Schen.* & Argentina: Prov. Jujuy, 25.02.1936. West 6114 (MO5257197) \\
\hline 35. & B. multiflora (L.f.) Mirb.* & $\begin{array}{l}\text { Costa Rica: Cartago, Paraiso, 18.03.1994. Lepiz et al. } 217 \text { (MO5593431) } \\
\text { Ecuador: 28.09.1959. Barclay 9394 (MO3189902) } \\
\text { Costa Rica: Prov. De Puntarenus, Monteverde, Monteverde Cloud Forest Reserve, 11.04.1983. Feinsinger et al. } \\
667 \text { (MO3108601) } \\
\text { Ecuador: Imbabura, Cotacachi, 25.05.1991. Panafiel et al. } 90 \text { (MO4065292) } \\
\text { Colombia: 30.08.1957. H.G. Barclay 5295 (MO04923181) }\end{array}$ \\
\hline 36. & B. multipes Benth.* & Ecuador: Loja, 03.10.1981. Besse et al. 751 (MO4310504) \\
\hline 37. & B. nematocaulon Killip & $\begin{array}{l}\text { Peru: Depto. Huanuco, Prov. Huanuco. Dto. Chinchao, Loc. San de Carpish. 03.11.2001. Salinas } 260 \\
\text { (USM193748). }\end{array}$ \\
\hline 38. & B. obovata Herb.* & Nicaragua: Atlántico Sur, 13-14.02.1978. Vincelli 209 (MO2698155) \\
\hline 39. & B. ovata (Cov.) Mirb. & Peru: Depto. de Lima, Entre Matucana \& San Mateo, 17.01.1949. Ferreyra 5300 (MOL00007277) \\
\hline
\end{tabular}


Table 1. Continuation

\begin{tabular}{|c|c|c|}
\hline No. & Taxa & Voucher specimens \\
\hline 40. & B. pardina Herb. & Ecuador: Carchi, 31.07.1989. Werff \& Gudino 10794 (MO3805857) \\
\hline 41. & B. patacocensis Herb. & Ecuador: Imbabura, 08.11.1990. Luteyn et al. 14078 (MO5829477) \\
\hline 42. & B. patinii Baker ${ }^{*}$ & Colombia: Antioquia, Salgar, 29.09.1987. J.L. Zarucchi et al. 5934 (MO3592494) \\
\hline 43. & B. perglabra Harling \& Neuendorf* & Ecuador: Cotopaxi, 26.04.1979 Løjtnant \& Ulf Molau 13708 (MO2982422) \\
\hline 44. & B. peruviana Hofreiter* & Peru: Cajamarca, Contumaza, 31.01.1985. Stein \& Todzia 2033B (MO2384243) \\
\hline 45. & B. purpurea (R. \& P.) Herb. & Peru: Amazonas, Chachapoyas, 21.02.1984. Smith 6118 (MO3473926) \\
\hline 46. & B. rosea (R. \& P.) Herb.* & Bolivia: Santa Cruz, Manuel Maria Caballero, 10.04.2004. Ledezma et al. 710 (MO04829598) \\
\hline 47. & B. salsilla (L.) Mirb. & Chile: no. date.1914. Calvert s.n. (MO1612096) \\
\hline 48. & B. setacea (R. \& P.) Herb. & $\begin{array}{l}\text { Ecuador: Loja, 09.12.1994. Jorgensen et al. } 1373 \text { (MO04833026) } \\
\text { Peru: Depto. Amazonas, Prov. Chachapoyas, } 5.2-2.4 .97 \text {. Weigend et al. } 97 / 405 \text { (USM971405) }\end{array}$ \\
\hline 49. & B. suberecta Gereau* & Costa Rica: Limon, Cordillera de Talamanca, 11.09.1984. Devidse et al. 28957 (MO3211739) \\
\hline 50. & B. superba Herb.* & $\begin{array}{l}\text { Peru: Depto. Cuzco, Prov. Calca, Dto. Lares, Rayampata, Bosque Primario. 20.02.2005. Farfan et al. } 564 \\
\text { (USM00221035) }\end{array}$ \\
\hline 51. & B. tarmensis Kraenzl. & Peru: Junín, Chanchamayo, 08.03.1985. Stein \& Todzia 2350 (MO3294277) \\
\hline 52. & B. tribachiata Kraenzl. & $\begin{array}{l}\text { Peru: Depto. La Libertad, Prov. Otuzco, Road Otuzco-Usquil, } 05.02 \text { - 02.04.97. Weigend et al. 97/211b } \\
\text { (USM178661). }\end{array}$ \\
\hline 53. & B. trichophylla Killip* & Venezuela: Lara, 03.01.1979. Berry 3273 (MO2725720) \\
\hline 54. & B. weigendii Hofreiter \& E. Rodr.* & Peru: Depto. Ayacucho, Loc. Yanamonte, Aina. 20.12.1966. Tovar 5780 (USM161735) \\
\hline
\end{tabular}

The pollen parameters studied and the LM and SEM preparation methods used follow Sarwar et al. (2010). Measurements were taken from at least 30 randomly selected grains from each specimen (Tab. 2). To visualize the relationships among the studied species of the subgenera Baccata, Bomarea, Sphaerine, and Wichuraea, agglomerative hierarchical clustering (AHC) analyses were conducted using the XLSTAT 2009.3 program, based on the quantitative characters polar length $(\mathrm{P})$, equatorial diameter (E), $\mathrm{P} / \mathrm{E}$ ratio, and exine thickness. The palynological data for the subgenera Baccata, Sphaerine, and Wichuraea were taken from Sarwar et al. (2015), and dendrograms were built using AHC. Slides of all specimens have been deposited in the Hokkaido University Museum, Sapporo, Japan. Pollen size and shape classes were defined using the criteria of Erdtman (1952), and our descriptive terminology follows Punt et al. (2007) and Hesse et al. (2009). The sectional classification of subgenus Bomarea follows Harling \& Neuendorf (2003) and Hofreiter (2008).

\section{Results}

Based on LM and SEM observations, the Bomarea pollen grains studied were monad, large, ellipsoid (boat-shaped), heteropolar; monosulcate, sulcus on the convex part of the grain, distinct, long, straight, wide at the equator, narrow near the poles, sometimes extended to the proximal pole (Fig. 1A-D); auriculae-like structures at the end of the sulcus in B. acutifolia, B. amazonica, B. andreana (Croats 34872), B. bredemeyerana, B. cornigera, B. crocea, B. dispar, B. eu- ryphylla, B. formosissima, B. hirsuta, B. lutea, B. macusanii, B. multiflora (Barclay 5295), B. suberecta, B. multipes, B. martiana, B. nematocaulon, B. pardina, and B. tarmensis (Fig. 1E-F; Tab. 2); opercula-like structures present on the sulcus in B. ovata (Fig. 1D). Symmetry is bilateral. Size ranges from $25.26-38.82 \mu \mathrm{m}$ (polar length $\mathrm{P}$ ) $\times$ 47.18-69.30 $\mu \mathrm{m}$ (equatorial diameter E), $\mathrm{P} / \mathrm{E} 0.43-0.62$, peroblate to oblate in shape, exine thickness 1.11-2.76 $\mu \mathrm{m}$ (Tab. 2).

The pollen grains of Bomarea species were characterized by a semitectate exine sculpture. Four different exine sculpture types were observed:

Type I - Microreticulate (Figs. 1G-O, 2A-F; Tab. 2), with perforate muri, heterobrochate; lumina less than or equal to $1 \mu \mathrm{m}$ in length or diameter; observed in B. amazonica, B. andreana (Davidson 7239), B. aurantiaca (Hawkes et al. 4438), B. boliviensis, B. cornigera, B. crassifolia, B. dispar, B. edulis, $B$. endotrachys, $B$. hartwegii, $B$. hirtella, $B$. martiana, B. multiflora (Feinsinger et al.667), B. multipes, B. rosea, B. setacea (Jorgensen et al. 1373), B. suberecta, and B. peruviana.

Type II - Reticulate (Figs. 2G-O, 3A-O, 4A-F; Tab. 2), with perforate muri, heterobrochate; lumina larger than $1 \mu \mathrm{m}$ in length or diameter; observed in B. acutifolia, B. anceps, B. andreana (Croats 34872), B. angulata, B. bredemeyerana, B. campylophylla, B. carderi, B. chiriquina, B. cochabambensis, B. cordifolia, B. cornuta, B. costaricensis, B. crocea, B. euryphylla, B. formosissima, B. goniocaulon, B. hirsuta, B. lopezii, B. lutea, B. macusanii, B. multiflora (Barclay 9394; Lepiz 217; Panafiel et al. 90; Barclay 5295), B. nematocaulon, B. ovata, B. patacocensis, B. patinii, B. perglabra, B. purpurea, and B. trichophylla. 
Table 2. Variation in pollen characters of Bomarea subg. Bomarea showing mean value in micrometer and standard deviation. Minimum - maximum values in micrometer in parenthesis. Taxa are arranged alphabetically within the group. (A) Pollen grains with auriculae-like structures; n.d. Not discern.

\begin{tabular}{|c|c|c|c|c|c|c|}
\hline Name of Taxa & Polar length $(\mathrm{P})$ & Equatorial diameter $(\mathrm{E})$ & $\mathrm{P} / \mathrm{E}$ & Exine thickness & Exine sculpture ${ }^{*}$ & Fig. No. \\
\hline \multicolumn{7}{|l|}{ Bomarea subg. Bomarea (c. 79 spp.) } \\
\hline \multicolumn{7}{|l|}{ Section Multiflorae } \\
\hline B. acutifolia (A) & $32.41 \pm 1.81(28.29-37.30)$ & $56.48 \pm 1.99(53.08-60.33)$ & 0.57 & $1.43 \pm 0.11(1.20-1.60)$ & Type II & $2 \mathrm{G}$ \\
\hline B. amazonica (A) & $28.64 \pm 2.37(24.67-34.92)$ & $58.01 \pm 2.94(49.96-63.56)$ & 0.49 & $1.11 \pm 0.12(0.95-1.34)$ & Type I & $1 \mathrm{G}$ \\
\hline \multirow[t]{2}{*}{ B. andreana } & $33.26 \pm 3.92(27.92-39.65)$ & $59.89 \pm 5.06(48.83-63.95)$ & 0.56 & $1.16 \pm 0.29(0.85-1.69)$ & Type I & $1 \mathrm{H}$ \\
\hline & $38.35 \pm 2.88(33.14-43.39)$ & $66.65 \pm 3.51(55.71-73.56)$ & 0.58 & $1.40 \pm 0.10(1.20-1.57)$ & Type II & $2 \mathrm{H}$ \\
\hline B. aurantiaca Hawkes et al. 4438 & $37.59 \pm 2.91(31.38-42.03)$ & $67.32 \pm 3.03(60.79-73.21)$ & 0.56 & $1.50 \pm 0.21(0.95-1.90)$ & Type III & - \\
\hline Cano 4679 & n.d. & n.d. & n.d. & n.d. & Type III & $4 \mathrm{G}$ \\
\hline B. bredemeyerana (A) & $31.58 \pm 1.61(28.28-34.94)$ & $59.05 \pm 2.53(54.57-65.30)$ & 0.53 & $1.40 \pm 0.19(0.93-1.60)$ & Type II & $2 \mathrm{I}$ \\
\hline B. chiriquina & $36.22 \pm 2.29(31.41-40.45)$ & $58.41 \pm 3.21(52.68-63.40)$ & 0.62 & $1.26 \pm 0.14(0.95-1.53)$ & Type II & $2 J$ \\
\hline B. costaricensis & n.d. & n.d. & n.d. & n.d. & Type II & $2 \mathrm{~K}$ \\
\hline B. crassifolia & $34.51 \pm 2.50(29.87-40.43)$ & $59.74 \pm 3.20(53.07-66.35)$ & 0.58 & $1.59 \pm 0.09(1.45-1.80)$ & Type I & $1 \mathrm{I}$ \\
\hline B. crocea (A) & $32.26 \pm 1.26(30.22-35.57)$ & $52.89 \pm 3.19(41.17-56.90)$ & 0.61 & $1.18 \pm 0.08(1.04-1.35)$ & Type II & $2 \mathrm{~L}$ \\
\hline B. densiflora $\mathrm{V}$ & $33.60 \pm 4.05(31.12-38.27)$ & $60.00 \pm 1.83(57.89-61.10)$ & 0.56 & $1.79 \pm 0.04(1.75-1.82)$ & - & - \\
\hline \multirow[t]{2}{*}{ Vigo 6229} & $25.26 \pm 3.06(21.90-32.81)$ & $47.18 \pm 3.70(39.83-53.85)$ & 0.54 & $1.37 \pm 0.18(1.15-1.80)$ & Type IV & $4 \mathrm{~J}$ \\
\hline & $30.87 \pm 2.42(26.48-35.42)$ & $49.83 \pm 2.93(44.84-56.65)$ & 0.62 & $1.34 \pm 0.13(1.10-1.62)$ & Type I & - \\
\hline B. euryphylla (A) & $32.86 \pm 2.83(26.86-37.46)$ & $59.61 \pm 2.91(53.47-66.76)$ & 0.55 & $1.80 \pm 0.15(1.53-2.12)$ & Type II & $2 \mathrm{M}$ \\
\hline B. formosissima (A) & $31.53 \pm 1.84(28.77-35.64)$ & $58.32 \pm 2.29(53.75-63.66)$ & 0.54 & $1.67 \pm 0.14(1.50-2.05)$ & Type II & $2 \mathrm{~N}$ \\
\hline B. hartwegii & $31.69 \pm 2.57(26.54-37.95)$ & $58.60 \pm 2.77(52.77-62.97)$ & 0.54 & $1.64 \pm 0.14(1.43-1.94)$ & Type I & $1 \mathrm{~J}$ \\
\hline B. hirsuta (A) & $31.53 \pm 2.65(26.48-37.95)$ & $59.05 \pm 2.54(50.90-63.67)$ & 0.53 & $1.57 \pm 0.13(1.30-1.75)$ & Type II & $2 \mathrm{O}$ \\
\hline B. lutea (A) & $35.84 \pm 2.28(31.80-41.12)$ & $69.30 \pm 3.41(61.10-76.48)$ & 0.52 & $1.89 \pm 0.15(1.53-2.18)$ & Type II & $3 \mathrm{~A}$ \\
\hline B. macusanii (A) & $35.59 \pm 1.86(31.86-39.75)$ & $59.02 \pm 2.53(51.15-61.82)$ & 0.60 & $1.55 \pm 0.11(1.34-1.75)$ & Type II & $3 B$ \\
\hline \multirow[t]{4}{*}{ B. multiflora } & $34.99 \pm 2.60(30.99-40.57)$ & $63.22 \pm 3.21(55.63-72.46)$ & 0.55 & $1.73 \pm 0.15(1.53-2.16)$ & Type II & $3 \mathrm{C}$ \\
\hline & $31.83 \pm 3.24(28.08-43.47)$ & $58.39 \pm 3.02(52.44-65.11)$ & 0.55 & $1.26 \pm 0.15(0.95-1.60)$ & Type II & $3 \mathrm{D}$ \\
\hline & $34.08 \pm 2.02(30.47-38.91)$ & $58.28 \pm 2.70(53.27-63.18)$ & 0.58 & $1.54 \pm 0.07(1.43-1.75)$ & Type I & $1 \mathrm{~K}$ \\
\hline & n.d. & n.d. & n.d. & n.d. & Type II & $3 \mathrm{E}$ \\
\hline Barclay 5295 (A) & $33.72 \pm 2.63(29.23-40.15)$ & $62.43 \pm 3.15(57.50-68.51)$ & 0.54 & $1.80 \pm 0.18(1.53-2.16)$ & Type II & $3 \mathrm{~F}$ \\
\hline B. patacocensis & $32.15 \pm 3.70(26.12-40.47)$ & $63.26 \pm 2.99(56.76-68.00)$ & 0.51 & $1.63 \pm 0.17(1.27-1.92)$ & Type II & $3 \mathrm{G}$ \\
\hline B. patinii & $31.78 \pm 2.11(27.71-35.95)$ & $59.84 \pm 3.59(51.66-65.92)$ & 0.53 & $1.82 \pm 0.13(1.53-2.12)$ & Type II & $3 \mathrm{H}$ \\
\hline B. purpurea & $29.51 \pm 2.49(26.37-34.23)$ & $57.82 \pm 2.41(54.31-60.71)$ & 0.51 & $1.31 \pm 0.21(1.02-1.57)$ & - & - \\
\hline B. setacea Jorgensen et al. 1373 & n.d. & n.d. & n.d. & n.d. & Type I & - \\
\hline Weigend et al. 97/405 & $31.01 \pm 2.07(27.61-36.04)$ & $51.35 \pm 2.60(46.96-57.05)$ & 0.60 & $1.64 \pm 0.17(1.43-2.16)$ & Type IV & $4 \mathrm{~K}$ \\
\hline B. suberecta (A) & $27.80 \pm 1.51(24.25-30.87)$ & $53.31 \pm 1.84(49.36-56.62)$ & 0.52 & $1.51 \pm 0.18(1.25-1.80)$ & Type I & $1 \mathrm{~L}$ \\
\hline B. superba & $38.03 \pm 3.35(33.44-43.39)$ & $65.18 \pm 3.76(60.80-71.94)$ & 0.58 & $1.72 \pm 0.20(1.53-2.12)$ & Type IV & $4 \mathrm{~L}$ \\
\hline Section Edules & & & & & & \\
\hline B. campylophylla & $38.82 \pm 3.06(32.26-45.50)$ & $67.55 \pm 3.19(61.54-74.27)$ & 0.57 & $1.55 \pm 0.09(1.43-1.75)$ & Type II & $3 \mathrm{I}$ \\
\hline B. cordifolia & $34.30 \pm 2.67(28.17-41.03)$ & $61.48 \pm 2.39(57.10-67.62)$ & 0.56 & $1.65 \pm 0.11(1.45-1.83)$ & Type II & $3 \mathrm{~J}$ \\
\hline B. cornigera $(\mathrm{A})$ & $30.64 \pm 1.84(26.54-34.06)$ & $55.17 \pm 2.30(51.52-60.60)$ & 0.56 & $1.52 \pm 0.18(1.15-1.80)$ & Type I & $1 \mathrm{M}$ \\
\hline B. cornuta & $29.15 \pm 2.08(24.78-32.63)$ & $54.29 \pm 2.04(49.96-57.37)$ & 0.54 & $1.25 \pm 0.10(1.04-1.53)$ & Type II & $3 \mathrm{~K}$ \\
\hline B. dolichocarpa & $28.05 \pm 3.08(24.41-33.10)$ & $65.35 \pm 5.76(55.47-74.06)$ & 0.43 & $2.76 \pm 0.48(2.12-3.66)$ & - & \\
\hline B. edulis & n.d. & n.d. & n.d. & n.d. & Type I & $1 \mathrm{~N}$ \\
\hline B. lopezii & $35.94 \pm 2.33(31.78-39.79)$ & $59.56 \pm 2.83(52.72-65.39)$ & 0.60 & $1.37 \pm 0.19(1.07-1.75)$ & Type II & $3 \mathrm{~L}$ \\
\hline B. obovata & n.d. & n.d. & n.d. & n.d. & Type IV & $4 \mathrm{M}$ \\
\hline B. ovata & $30.14 \pm 3.67(22.39-35.87)$ & $59.66 \pm 4.09(52.84-66.87)$ & 0.51 & $1.42 \pm 0.19(1.20-1.90)$ & Type II & $3 \mathrm{M}$ \\
\hline
\end{tabular}


Table 2. Continuation.

\begin{tabular}{|c|c|c|c|c|c|c|}
\hline Name of Taxa & Polar length $(\mathrm{P})$ & Equatorial diameter $(\mathrm{E})$ & $\mathrm{P} / \mathrm{E}$ & Exine thickness & Exine sculpture $^{*}$ & Fig. No \\
\hline \multicolumn{7}{|l|}{ Section Goniocaulon } \\
\hline B. angulata & n.d. & n.d. & n.d. & n.d. & Type II & $3 \mathrm{~N}$ \\
\hline B. goniocaulon & $32.26 \pm 2.61(27.99-38.27)$ & $59.31 \pm 3.22(51.81-66.50)$ & 0.54 & $2.12 \pm 0.31(1.75-2.80)$ & Type II & 30 \\
\hline B. multiples (A) & $32.69 \pm 1.63(28.89-36.28)$ & $61.23 \pm 2.37(55.84-66.18)$ & 0.53 & $1.81 \pm 0.18(1.53-2.18)$ & Type I & 10 \\
\hline B. perglabra & $35.11 \pm 3.17(31.16-40.67)$ & $60.11 \pm 3.75(54.52-64.25)$ & 0.58 & $1.40 \pm 0.15(1.20-1.56)$ & Type II & $4 \mathrm{~A}$ \\
\hline \multicolumn{7}{|l|}{ Section Pardinae } \\
\hline B. pardina $(\mathrm{A})$ & n.d. & n.d. & n.d. & n.d. & Type III & $4 \mathrm{H}$ \\
\hline \multicolumn{7}{|c|}{ Don't fit into any section } \\
\hline B. anceps & $30.87 \pm 2.00(27.61-33.96)$ & $57.45 \pm 2.21(52.20-61.34)$ & 0.54 & $1.63 \pm 0.16(1.34-2.09)$ & Type II & $4 \mathrm{~B}$ \\
\hline B. boliviensis & n.d. & n.d. & n.d. & n.d. & Type I & $2 \mathrm{~A}$ \\
\hline B. cochabambensis & $29.40 \pm 3.60(24.73-37.61)$ & $55.16 \pm 3.62(48.78-61.77)$ & 0.53 & $1.12 \pm 0.11(0.95-1.27)$ & Type II & $4 \mathrm{C}$ \\
\hline B. dispar (A) & $32.56 \pm 2.43(28.30-38.40)$ & $58.76 \pm 3.08(51.15-65.13)$ & 0.54 & $1.63 \pm 0.13(1.43-1.90)$ & Type I & $2 \mathrm{~B}$ \\
\hline B. hirtella & $36.03 \pm 2.78(30.97-41.79)$ & $67.80 \pm 3.07(62.30-73.63)$ & 0.53 & $1.85 \pm 0.20(1.57-2.40)$ & Type I & $2 \mathrm{C}$ \\
\hline B. martiana (A) & $32.43 \pm 3.29(28.17-38.63)$ & $56.39 \pm 4.52(48.51-68.87)$ & 0.58 & $1.38 \pm 0.19(1.20-1.90)$ & Type I & $2 \mathrm{D}$ \\
\hline B. nematocaulon (A) & $27.88 \pm 2.23(24.25-34.51)$ & $51.18 \pm 2.97(44.42-57.64)$ & 0.55 & $1.58 \pm 0.13(1.27-1.80)$ & Type II & $4 \mathrm{D}$ \\
\hline B. rosea & $34.66 \pm 1.84(31.57-38.25)$ & $67.60 \pm 2.95(61.99-75.14)$ & 0.51 & $2.13 \pm 0.22(1.75-2.58)$ & Type I & $2 \mathrm{E}$ \\
\hline B. tarmensis (A) & $27.93 \pm 2.98(23.59-31.40)$ & $56.37 \pm 3.16(53.17-59.76)$ & 0.50 & $1.82 \pm 0.19(1.64-2.12)$ & Type IV & $4 \mathrm{~N}-\mathrm{O}$ \\
\hline B. trichophylla & $32.13 \pm 1.80(28.74-35.41)$ & $54.11 \pm 2.44(51.21-59.00)$ & 0.59 & $1.76 \pm 0.16(1.53-2.12)$ & Type II & $4 \mathrm{E}$ \\
\hline B. weigendii & n.d. & n.d. & n.d. & n.d. & Type III & $4 \mathrm{I}$ \\
\hline \multicolumn{7}{|c|}{ Bomarea subg. Baccata (5 spp.) } \\
\hline B. carderi & $36.39 \pm 2.63(31.57-42.80)$ & $60.77 \pm 2.50(55.96-66.14)$ & 0.60 & $1.34 \pm 0.14(0.95-1.57)$ & Type II & $4 \mathrm{~F}$ \\
\hline \multicolumn{7}{|c|}{ Bomarea subg. Wichurea (16 spp.) } \\
\hline B. peruviana & $30.00 \pm 3.74(25.88-38.07)$ & $61.29 \pm 4.45(54.15-68.71)$ & 0.49 & $1.82 \pm 0.20(1.53-2.12)$ & Type I & $2 \mathrm{~F}$ \\
\hline
\end{tabular}

* Exine ornamentation type by SEM. Type I, microreticulate, lumina less than or equal $1 \mu \mathrm{m}$ in size; Type II, reticulate, lumina larger than $1 \mu \mathrm{m}$ in size; Type III, coarsely rugulate; Type IV, psilate-perforate. For details see text.

Type III - Coarsely rugulate (Fig. 4G-I; Tab. 2); observed in B. aurantiaca (Cano 4679), B. pardina, and B. weigendii. Type IV - Psilate-perforate (Fig. 4J-O; Tab. 2); observed in B. densiflora (Vigo 6229), B. obovata, B. setacea (Weigend et al. 97/405), B. superba, and B. tarmensis.

Granula were sometimes visible at the bottom of the lumina; these may correspond to "free standing columellae" (e.g. Fig. 1G; Hesse et al. 2009). The exine sculpture along with sulci was similar to that appearing at the equatorial position, but had relatively smaller lumina.

In agglomerative hierarchical clustering (AHC) analyses of the genus Bomarea using quantitative pollen data, the species studied were distributed in either two (similarity-based) or four (dissimilarity-based) major clusters (Fig. 5). Among these clusters, three include at least one species from each subgenus; cluster 1 (blue) comprises most of the taxa studied (41), cluster 2 (pink) comprises 12 taxa, cluster 3 (brown) comprises 16 taxa, and cluster 4 (black) comprises the single species B. ampayesana from the subgenus Wichuraea.

\section{Discussion}

The Bomarea species studied were stenopalynous and characterized by monad, monosulcate, large pollen grains (Figs. 1-4; Tab. 2; Sarwar et al. 2015). The pollen morphology of 36 of these species, indicated by asterisks in Tab. 1, was studied for the first time using either LM or SEM. Our results are in agreement with previous reports (Erdtman 1952; Heusser 1971; Neuendorf 1977; Schulze 1978; Kosenko 1994; Sanso \& Xifreda 2001; Alzate 2007; Sarwar et al. 2015). The monophyly of the genus Bomarea is supported by molecular data (Aagesen \& Sanso 2003; Alzate et al. 2008) and by the palynological data as well (Tab. 2; Sarwar et al. 2015). However, there were significant differences in the values of the quantitative palynological characters, which may to some extent be related to differences in the mounting media (Meltsov et al. 2008) and the methods of preparation of the pollen grains (Schols et al. 2004), as well as the species' geographical distribution, floral size, etc. (AKM Golam Sarwar pers. obs.). For example, there were 
A

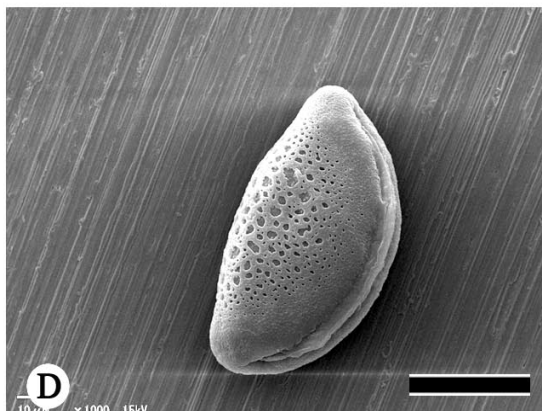

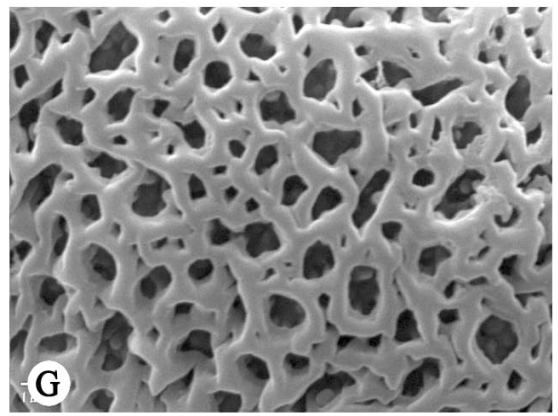
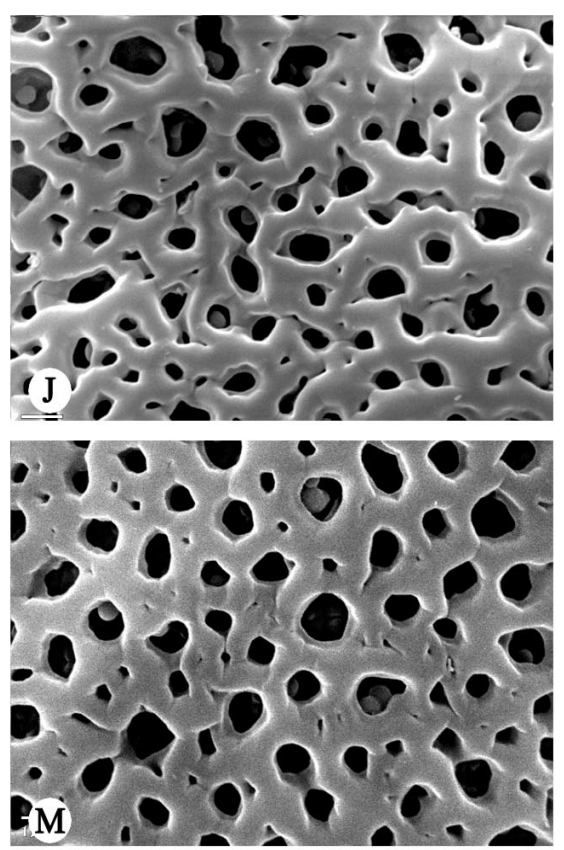

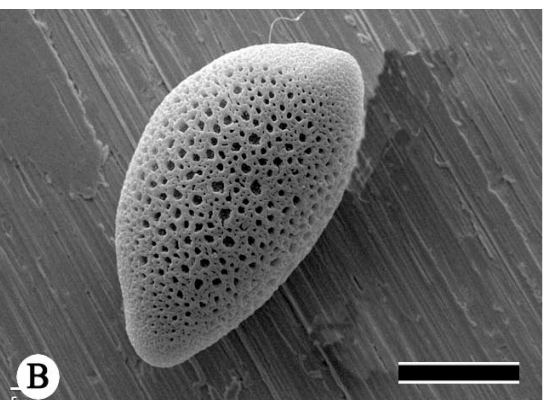

(C)
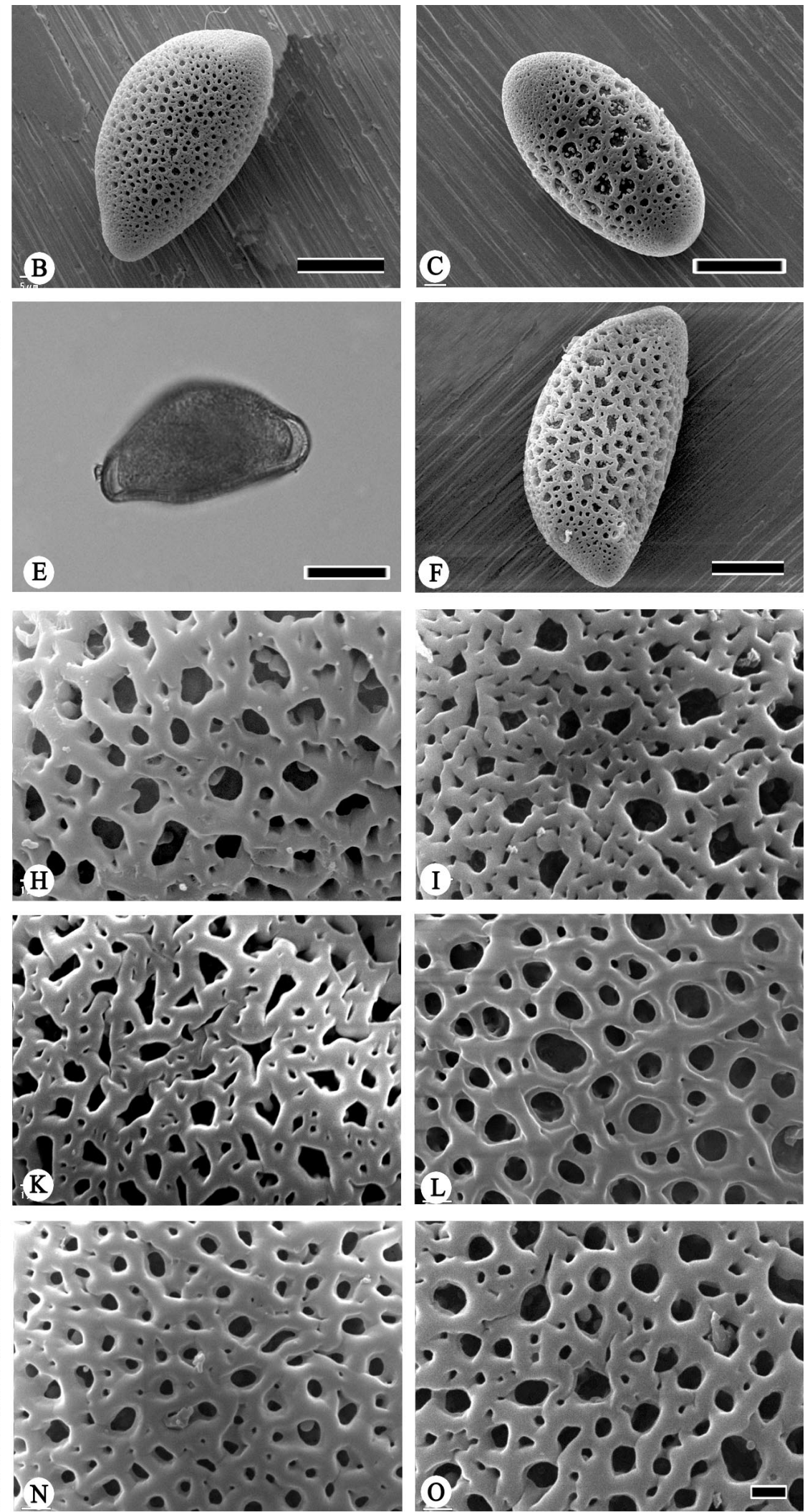

Figure 1. Light and scanning electron micrographs of Bomarea pollen. Pollen grains (A-F); exine sculpture (G-O). A. B. rosea (Ledezma et al. 710); B. B. crocea (Galiano et al. 6987); C. B. cochabambensis (Cutler 7678); D. B. ovata (Ferreyra 5300); E. B. multiflora (Barclay 5295); F. B. andreana (Croats 34872); G. B. amazonica (Barbour 2859); H. B. andreana (Davidson 7239); I. B. crassifolia (Fosberg 27845); J. B. hartwegii (Campos et al. 5735); K. B. multiflora (Feinsinger et al. 667); L. B. suberecta (Devidse et al. 28957); M. B. cornigera (Stein \& Todzia 2230); N. B. edulis (Sarwar \& Hoshino s. n.); O. B. multipes (Besse et al. 751). Scale bars: A-F, 10 $\mu \mathrm{m} ; \mathrm{G}-\mathrm{O}, 1 \mu \mathrm{m}$. Specimen numbers are detailed in Tab. 1. 


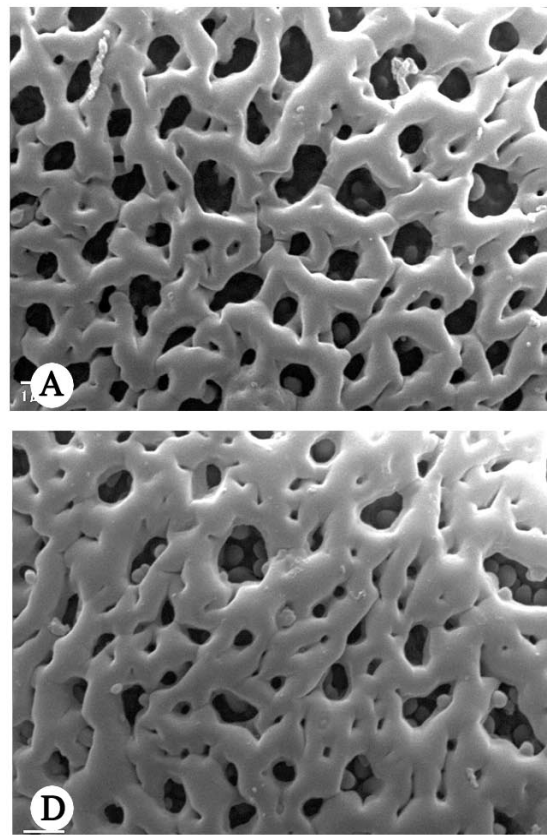

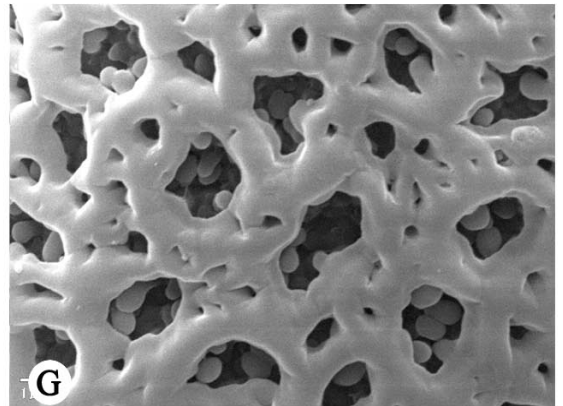
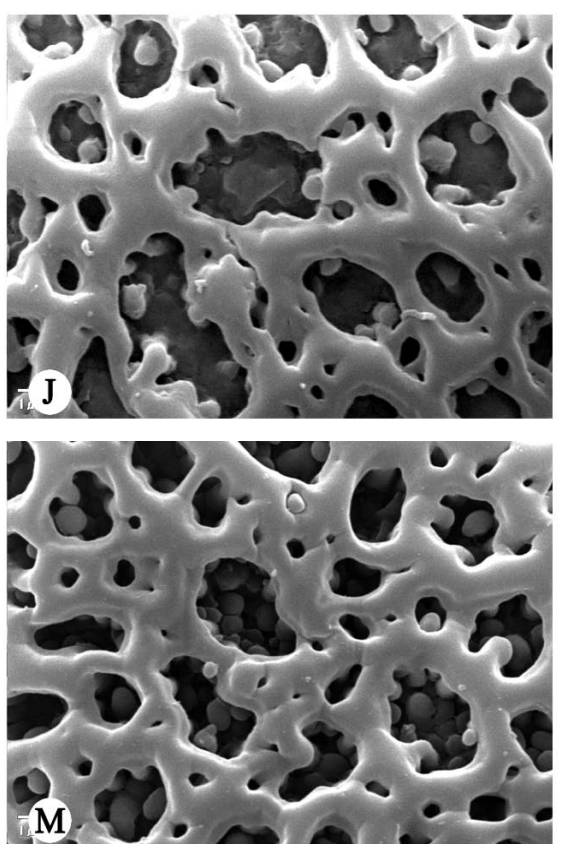

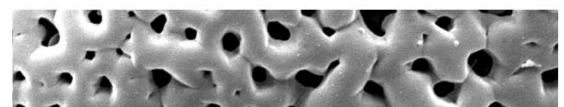
$\rightarrow a x$ a a

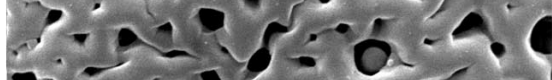

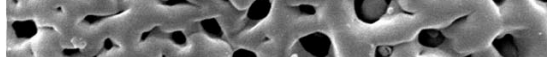

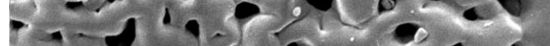
is $\left.-a^{2}+202 \cdot\right)$,

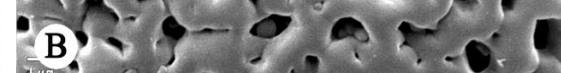
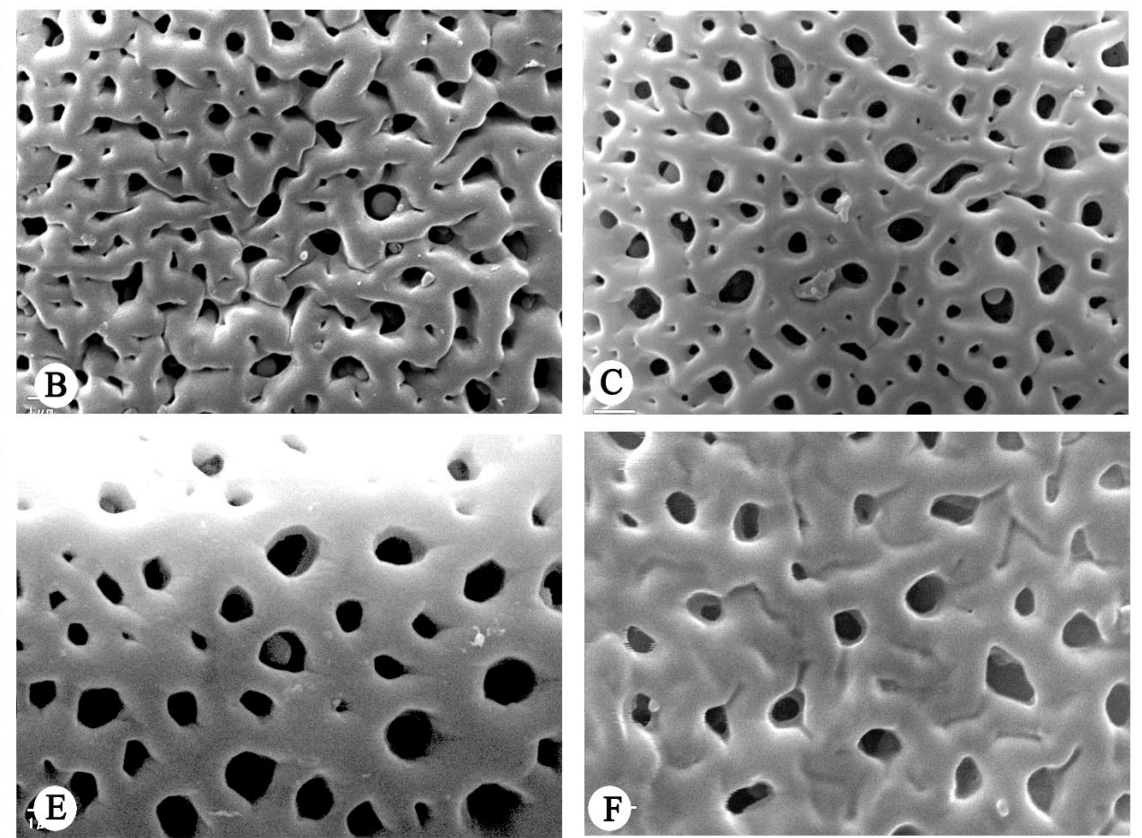

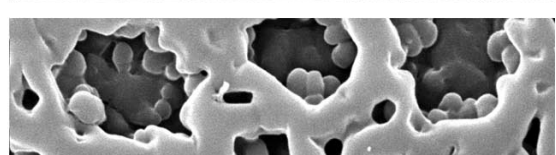
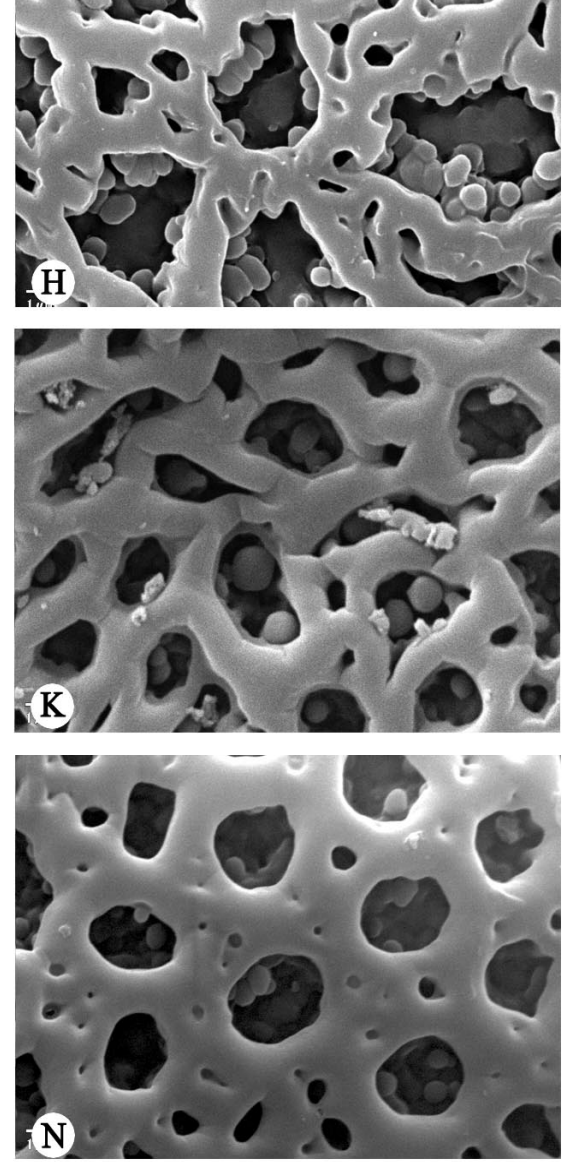
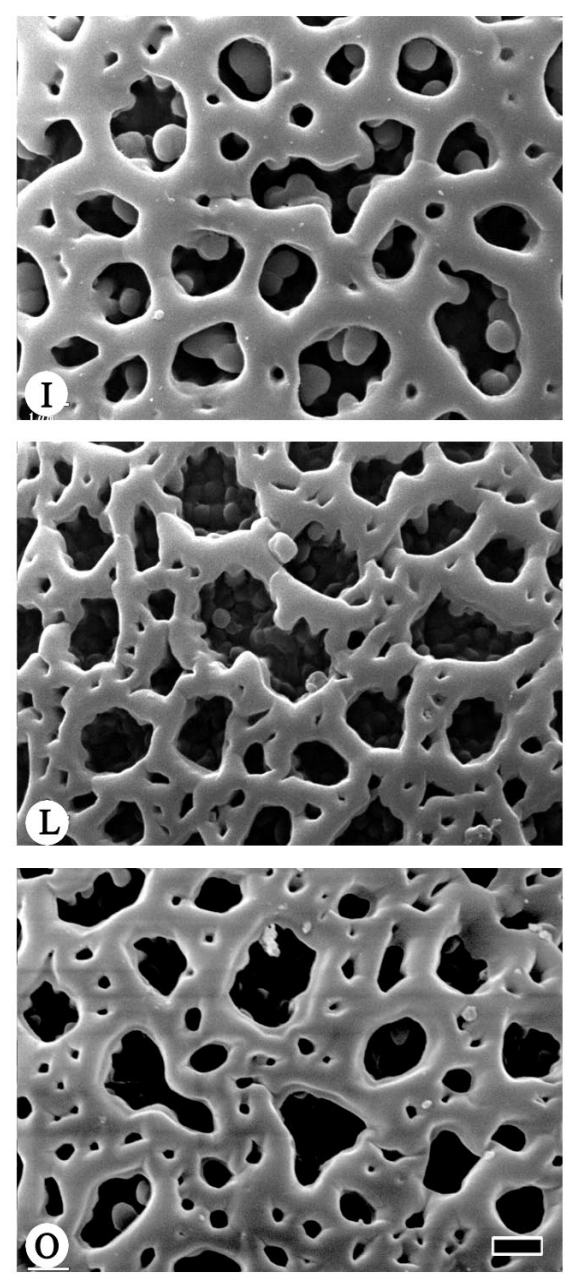

Figure 2. Scanning electron micrographs of Bomarea pollen. Exine sculpture (A-O). A. B. boliviensis (Wood 7818); B. B. dispar (Smith 6582); C. B. hirtella (Chagala 68); D. B. martiana (West 6114); E. B. rosea (Ledezma et al. 710); F. B. peruviana (Stein \& Todzia 2033B); G. B. acutifolia (Davidse 24682); H. B. andreana (Croats 34872); I. B. bredemeyerana (Liesner et al. 7935); J. B. chiriquina (Mori \& Kullunki 5651); K. B. costaricensis (Chavarria 663); L. B. crocea (Galiano et al. 6987); M. B. euryphylla (Palacios 6420); N. B. formosissima (Nunez et al. 8511); O. B. hirsuta (Chavarria 756). Scale bars: A-O, $1 \mu$ m. Specimen numbers are detailed in Tab. 1. 

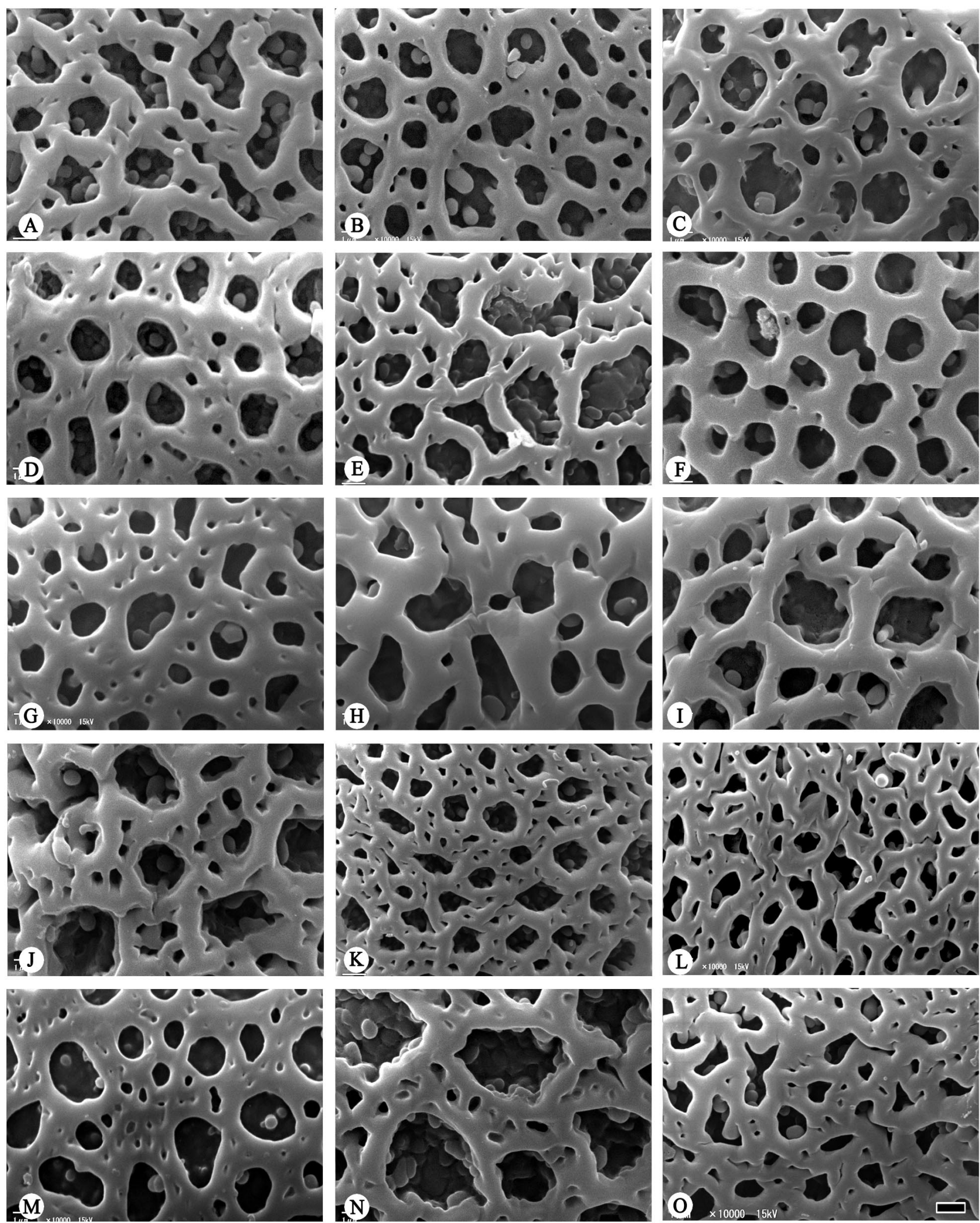

Figure 3. Scanning electron micrographs of Bomarea pollen. Exine sculpture (A-O). A. B. lutea (Scolnik 1448); B. B. macusanii (Nunez et al. 8477); C. B. multiflora (Lepiz 217); D. B. multiflora (Barclay 9394); E. B. multiflora (Panafiel et al. 90); F. B. multiflora (Barclay 5295); G. B. patacocensis (Luteyn et al. 14078); H. B. patinii (Zarucchi et al. 5934); I. B. campylophylla (Miranda et al. 588); J. B. cordifolia (Foster \& Smith 9076); K. B. cornuta (Stein \& Todzia 2275); L. B. lopezii (Sagastegui 11402); M. B. ovata (Ferreyra 5300); N. B. angulata (Sagastegui 12759); O. B. goniocaulon (Hamilton \& Holligan 522). Scale bars: A-O, $1 \mu \mathrm{m}$. Specimen numbers are detailed in Tab. 1. 

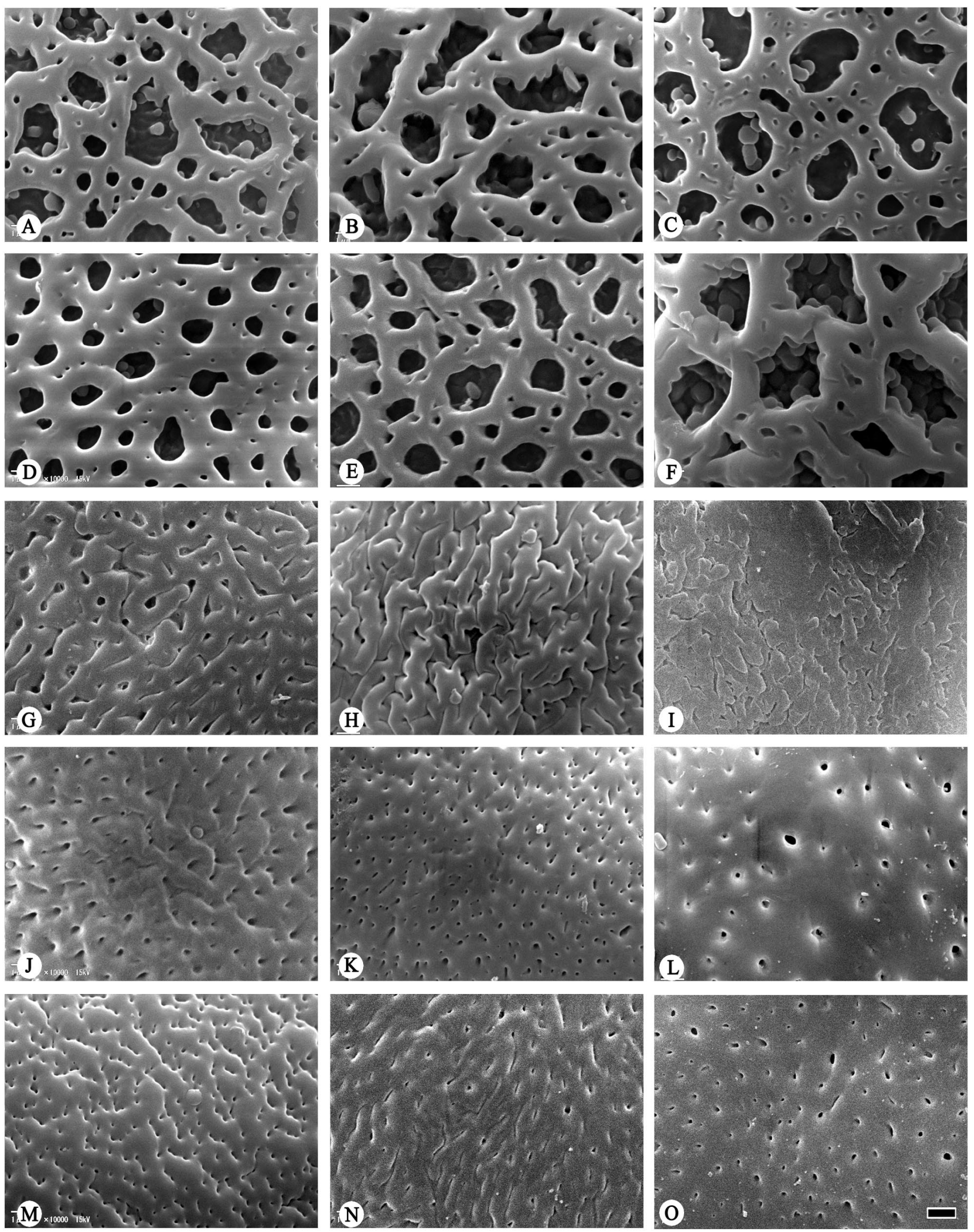

Figure 4. Scanning electron micrographs of Bomarea pollen. Exine sculpture (A-O). A. B. perglabra (Løjtnant \& Ulf Molau 13708); B. B. anceps (MOL2940370); C. B. cochabambensis (Cutler 7678); D. B. nematocaulon (Salinas 260); E. B. trichophylla (Berry 3273); F. B. carderi (Churchill 3904); G. B. aurantiaca (Cano 4679); H. B. pardina (Werff \& Gudino 10794); I. B. weigendii (Tovar 5780); J. B. densiflora (Vigo 6229); K. B. setacea (Weigend et al. 97/405); L. B. superba (Farfan et al. 564); M. B. obovata (Vincelli 209); N-O. B. tarmensis (Stein \& Todzia 2350). Scale bars: A-O, $1 \mu \mathrm{m}$. Specimen numbers are detailed in Tab. 1. 


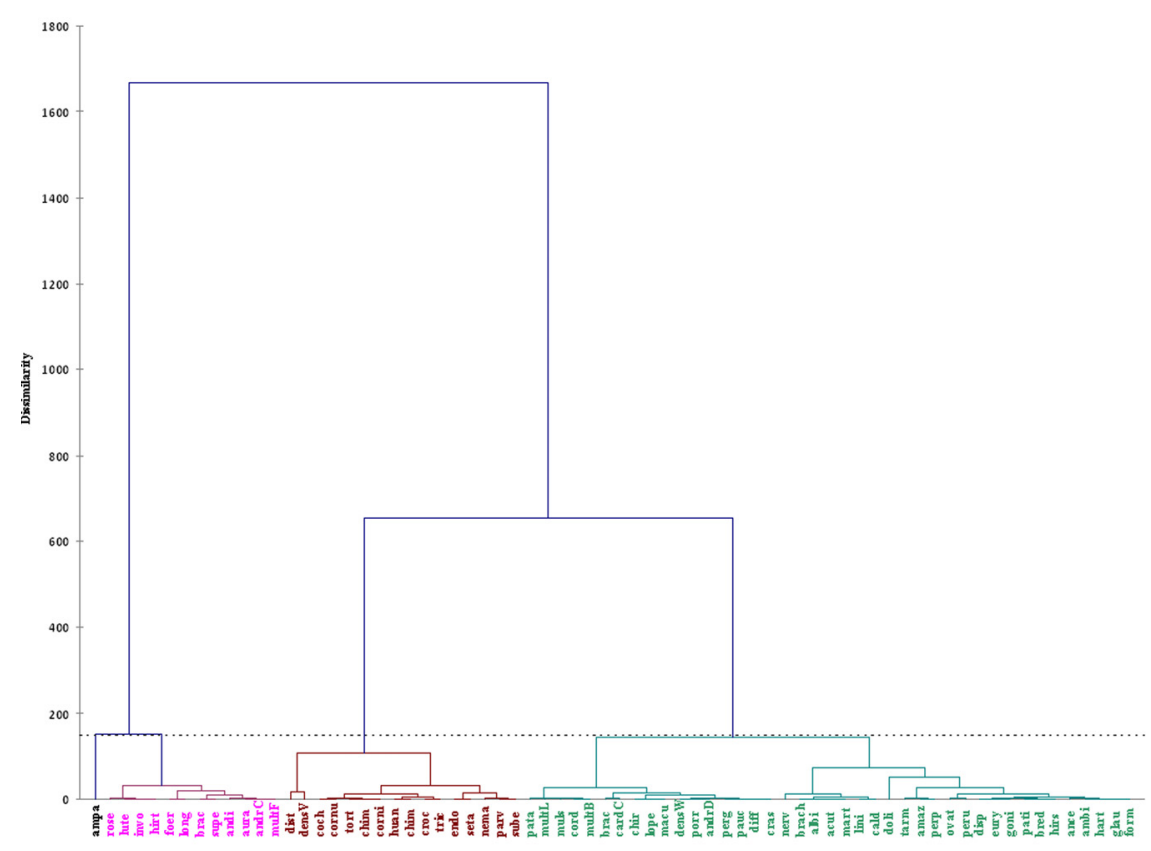

Figure 5. Dendrogram obtained from quantitative data by agglomerative hierarchical clustering analysis. Names of species are abbreviated to the first four to six letters of the specific epithets.

significant differences between two B. andreana specimens, one of which had smaller pollen grains with a thinner exine (Davidson 7239, collected from the warmer region of Costa Rica), while the other had larger pollen grains with a thicker exine (Croats 34872, collected from the cooler region of Panama) (Tabs. 1, 2).

A wide and generally continuous pattern of variation in $\mathrm{P}, \mathrm{E}, \mathrm{P} / \mathrm{E}$ ratio, and exine thickness was observed at both the infra- and inter-species level (Tab. 2). Among the sections of Bomarea subgenus Bomarea, section Goniocaulon produces pollen grains with relatively consistent $\mathrm{P} / \mathrm{E}$ ratios (0.53-0.58) (Tab. 2). Pollen grain size within a genus is influenced by internal (chromosome number and floral character) and external (temperature, mineral nutrition, and water conditions) factors (Stanley \& Linskens 1974). However, no correlation between ploidy level and palynological features was observed in Bomarea. The only tetraploid species of Bomarea, B. hirtella, produced pollen grains similar in size to or slightly smaller than the diploid species (Tab. 2; Cave 1967).

Auricula-like structures in pollen grains have been previously reported from only four Bomarea species, namely, B. brachysepala, B. bracteolata, B. glaucescens, and B. huanuco (Sarwar et al. 2015). In the present study, pollen with auricula-like structures was observed in 19 taxa (Fig. 1E; Tab. 2), including in B. ceratophora (Neuendorf 1977) in the subgenus Bomarea. These auricula-like structures may have some taxonomic importance for the genus Bomarea since they are relatively common in the subgenus Bomarea, but are completely absent in members of the southern group of the subgenus Wichuraea (Tab. 2; Sarwar et al. 2015). Auriculate pollen grains are not common among extant plant taxa, and diverse Cretaceous pollen-bearing auriculate appendages have been variously described as gymnospermous and monocotyledonous (Elsik 1974). As the term "auriculae" is only applicable to the spores' structure (Punt et al. 2007), the term "apex" has sometimes been used for these auriculalike pollen structures (see Martín et al. 2012 for detailed terminological discussion).

A recent molecular phylogenetic analysis of Bomarea (Alzate et al. 2008) identified three major clades, but none of them correspond to traditionally recognized subgeneric taxa (Hofreiter \& Tillich 2002). Only seven of the species we studied were included in that molecular analysis, and these seven are positioned in two different clades (Fig. 2 in Alzate et al. 2008). Exine sculpture was found to be the most important palynological character possessing phylogenetic importance. In the molecular phylogenetic analysis, four members of section Multiflorae (B. vestita syn. B. multiflora; Hofreiter 2008) were included in clade C; this was also supported and confirmed by palynological characters, especially exine sculpture Type II (Figs. 2I, O, 3C-F; Tab. 2). The other species of section Multiflorae (B. setacea) are sister to clade $B$ and are characterized by exine sculpture Type IV (Fig. 4K; Tab. 2), although wide variation was observed in the exine sculpture of two specimens of B. setacea (Type I $v$ s. Type IV; Tab. 2), which may be due to polymorphism. Bomarea setacea is polymorphous taxon, especially in its general stature and in the shape and size of the leaves and inflorescence (Harling \& Neuendorf 2003). Of the two other species of the subgenus Bomarea, B. edulis was included in clade A, and B. salsilla was sister to B. straminea (Fig. 2 in Alzate et al. 2008). 
Pollen grains of Bomarea species are characterized by their (micro-)reticulate exine sculptures (Figs. 1G-O, 2, 3, 4A-F; Sarwar et al. 2015), although coarsely rugulate and finely rugulate-perforate or psilate-perforate exine sculptures are also observed in a few Bomarea species (Fig. 4G-O; Hofreiter 2008; Sarwar et al. 2015). This may give additional support to the supposition that (micro-)reticulate exine sculptures might be a plesiomorphic character state for Bomarea and the coarsely rugulate and finely rugulateperforate or psilate-perforate exine sculptures might have evolved independently more than once (Alzate et al. 2008; Sarwar et al. 2015). These results may also confirm the major evolutionary trend in exine sculpture in the family Alstroemeriaceae from reticulate through rugulate-psilate to striate-reticulate, or vice-versa (Aagesen \& Sanso 2003; Sarwar et al. 2010; 2015).

Among the quantitative pollen characters analyzed, equatorial diameter had the greatest influence on the position of taxa in the AHC cladogram (Fig. 5; Tab. 2; Sarwar et al. 2015). The members of cluster 1 commonly produced pollen grains $52.67-62.93 \mu \mathrm{m}$ in size; cluster 2 produced relatively larger pollen grains $(63.93-69.30 \mu \mathrm{m})$; cluster 3 produced the smallest pollen grains $(44.46-55.30 \mu \mathrm{m})$. The large pollen grains of $B$. ampayesana (subgenus Wichuraea) might be one of the reasons for its very distinct position in cluster 4 (black) of the AHC (Fig. 5; Tab. 2; Sarwar et al. 2015). The greatest variation in pollen morphological features was observed in the subgenus Wichuraea (Fig. 5; Hofreiter \& Tillich 2002), which also exhibited the greatest variation in other morphological features.

There were significant differences in the species composition of the different subclades/clusters recovered from both the molecular phylogenetic analysis (based on a few species; Alzate et al. 2008) and the AHC (based on quantitative pollen features; Fig. 5). Moreover, analyses based on different genomes (nuclear $v s$. plastid) not only lead to disagreements in the evolutionary relationships of taxa, but also greatly affected other phylogeny-based inferences and interpretations relating to taxonomy, morphological evolution, historical biogeography, and phylogenetic diversity (Zhang et al. 2015). Therefore, the most reliable infrageneric classification of Bomarea will be achieved through combined analyses of morphological, palynological, and molecular data from more extentive sampling- of all the species in the genus.

\section{Acknowledgements}

We thank the directors and curators of the consulted herbaria for allowing us to examine or borrow specimens of polliniferous material. The first author is especially grateful to the Japan Society for the Promotion of Science (JSPS) for a Postdoctoral Fellowship for Foreign Researchers during the period of this study.

\section{References}

Aagesen L, Sanso M. 2003. The phylogeny of the Alstroemeriaceae based on morphology, rps 16 intron, and $r b c L$ sequence data. Systematic Botany 28: 47-69.

Alzate F. 2005. Three new species of Bomarea (Alstroemeriaceae) from the Andean region of Colombia. Novon 15: 253-258.

Alzate F. 2007. Two new species of Bomarea (Alstroemeriaceae) from Colombia. Novon 17: 141-144.

Alzate F, Mort ME, Ramirez M. 2008. Phylogenetic analyses of Bomarea (Alstroemeriaceae) based on combined analyses of nrDNA ITS, $p s b A$ trnH, rpoB-trnC and matK sequences. Taxon 57: 853-862.

Baker JG. 1888. Handbook of Amaryllideae. London, George Bell \& Sons.

Cave MS. 1967. In: Documented chromosome numbers of plants. Madroño 19: $134-136$

Elsik WC. 1974. Fossil auriculate pollen. Pollen Spores 16: 507-533.

Erdtman G. 1952. Pollen morphology and plant taxonomy - Angiosperms. Leiden, E. J. Brill.

Harling G, Neuendorf M. 2003. Alstroemeriaceae. In: Harling G, Andersson L. (eds). Flora of Ecuador. Vol. 71. Stockholm, Göteborg University. p. 3-108.

Hesse M, Halbritter H, Zetter R, Weber M, Buchner R, Frosch-Radivo A, Ulrich S. 2009. Pollen terminology: an illustrated handbook. New York, Springer.

Heusser CJ. 1971. Pollen and spores of Chile. Tucson, University of Arizona Press.

Hofreiter A. 2008. A revision of Bomarea subgenus Bomarea s. str. section Multiflorae (Alstroemeriaceae). Systematic Botany 33: 661-684.

Hofreiter A, Tillich H-J. 2002. The delimitation, ecology, distribution and infrageneric subdivision of Bomarea Mirbel (Alstroemeriaceae). Feddes Repertorium 113: 528-544.

Killip EP. 1935. New species of Bomarea from the Andes. Journal of the Washington Academy of Science 25: 370-377.

Kosenko VN. 1994. Pollen morphology of the family Alstroemeriaceae. Botanischesky Zhurnal 79: 1-8.

Martín J, Raymúndez MB, Vallès J, Garnatje T, Raimúndez E. 2012. Palynological study of the Venezuelan species of the genus Hymenocallis (Amaryllidaceae). Plant Systematics and Evolution 298: 695-701.

Meltsov V, Poska A, Saar M. 2008. Pollen size in Carex: the effect of different chemical treatments and mounting media. Grana 47: 220-233.

Neuendorf M. 1977. Pardiniae, a new section of Bomarea (Alstroemeriaceae). Botanical Notiser 130: 55-60.

Punt W, Hoen PP, Blackmore S, Nilsson S, Le Thomas A. 2007. Glossary of pollen and spore terminology. Review of Palaeobotany and Palynology 143: 1-81.

Sanso AM, Xifreda CC. 2001. Generic delimitation between Alstroemeria and Bomarea (Alstroemeriaceae). Annals of Botany 88: 1057-1069.

Sarwar AKM Golam, Hoshino Y, Araki H. 2010. Pollen morphology and infrageneric classification of Alstroemeria L. (Alstroemeriaceae). Grana 49: 227-242.

Sarwar AKM Golam, Hoshino Y, Araki H. 2015. Pollen morphology and its taxonomic significance in the genus Bomarea Mirb. (Alstroemeriaceae) - I. Subgenera Baccata, Sphaerine, and Wichuraea. Acta Botanica Brasilica 29: 425-432.

Schols P, Es K, d'Hondt C, Merckx V, Smets E, Huysmans S. 2004. A new enzyme-based method for the treatment of fragile pollen grains collected from herbarium material. Taxon 53: 777-782.

Schulze W. 1978. Beitragezur Taxonomie der Liliifioren III. Alstroemeriaceae. Wissenschaftliche Zeitschrift der Friedrich-SchillerUniversitat Jena/Thüringen, Mathematisch-naturwissenschaftliche Reihe 27: 79-85.

Stanley RG, Linskens HF. 1974. Pollen: biology, biochemistry and management. Berlin, Springer-Verlag.

Thiers B. 2007. Index Herbariorum: a global directory of public herbaria and associated staff. New York Botanical Garden's virtual herbarium. http://sweetgum.nybg.org/ih/. 27 Jan. 2015.

Zhang Q, Feild TS, Antonelli A. 2015. Assessing the impact of phylogenetic incongruence on taxonomy, floral evolution, biogeographical history, and phylogenetic diversity. American Journal of Botany 102: 566-580. 